\title{
Utilidad de las secuencias potenciadas en susceptibilidad paramagnética (SWI) para diferenciar crisis somestésicas de accidentes isquémicos transitorios en un paciente con angiopatía amiloide cerebral
}

\section{The use of SWI-MRI to differentiate between seizures and transient ischemic attacks in a patient with cerebral amyloid angiopathy}

\author{
C. Gasca-Salas ${ }^{1}$, R. García de Eulate ${ }^{2}$, P. Pastor ${ }^{1,3}$
}

\section{RESUMEN}

Introducción. La angiopatía amiloide cerebral (AAC) se caracteriza por el depósito de $\beta$-amiloide en la pared de las arterias de pequeño y mediano calibre de la corteza cerebral y leptomeninges. La sintomatología puede incluir eventos transitorios de diverso origen, pudiendo ser difícil el diagnóstico diferencial en el momento agudo.

Caso clínico. Varón con antecedente de ictus isquémico, que presentó crisis somestésicas secundarias a microsangrado cerebral por AAC que simulaban ataques isquémicos transitorios. Se redujo la medicación antiagregante y posteriormente se inició tratamiento antiepiléptico desapareciendo los episodios. Las secuencias de susceptibilidad paramagnética en Resonancia Magnética cerebral fueron de gran utilidad para el diagnóstico (SWI-RM).

Conclusiones. Los microsangrados de la AAC pueden manifestarse en forma de crisis, simulando déficits focales sensitivos transitorios que pueden ser atribuidos erróneamente a isquemia cerebral. Este caso sugiere que, incluso si existe historia de accidentes cerebrovasculares isquémicos, deberían considerarse microsangrados por AAC como causa posible de pseudoaccidentes isquémicos transitorios. Las secuencias SWI-RM pueden ser útiles para identificarlos. De este modo puede prevenirse el inicio o escalada del tratamiento antiagregante o anticoagulante, evitando complicaciones potencialmente devastadoras.

Palabras clave. Accidentes isquémicos transitorios. Angiopatía amiloide cerebral. Crisis somestésicas. Microsangrados. Resonancia magnética cerebral.

\begin{abstract}
Background. Cerebral amyloid angiopathy (CAA) is characterized by the deposit of $\beta$-amyloid on the walls of small and medium-sized arteries of the cerebral cortex and leptomeninges causing cerebral bleeding. Clinical presentations may include transient neurological events for which differential diagnosis can be difficult.
\end{abstract}

Case report. We report a subject with a medical history of recent stroke who presented somesthetic seizures mimicking transient ischemic attacks owing to CAA microbleeding. Antiplatelet treatment was reduced and after lamotrigine was commenced the episodes disappeared. Susceptibility-weighted magnetic resonance imaging was very helpful for diagnosis (SWI-MRI).

Conclusions. CAA microbleeding can be manifested in the form of seizures mimicking focal transient sensitive neurological deficits that can be erroneously attributed to cerebral ischemia. The present case report suggests that, despite the presence of a past medical history of strokes, neurologists should consider CAA microbleeding as a possible cause of pseudo-transient ischemic attacks. High-resolution neuroimaging including SWIMRI imaging can be helpful in identifying cortical microbleedings. In this way, the start or increase of antiplatelet treatment can be avoided, and the risk of potentially fatal complications minimized.

Key words. Transient ischemic attacks. Cerebral amyloid angiopathy. Somesthetic seizures. Microbleeding. Magnetic Resonance.
1. Departamento de Neurología. Clínica Universidad de Navarra.

2. Departamento de Radiología. Clínica Universidad de Navarra.

3. Laboratorio de Neurogenética. Clínica Universidad de Navarra.

\section{Correspondencia}

Carmen Gasca Salas

Clínica Universidad de Navarra

Avda. Pío XII, 36

31008 Pamplona

E-mail. cgasca@unav.es

Recepción: 6 de abril de 2011

Aceptación provisional: 27 de abril de 2011

Aceptación definitiva: 3 de mayo de 2011 


\section{INTRODUCCIÓN}

La angiopatía amiloide cerebral (AAC) es una entidad neurológica causada por la acumulación de $\beta$ amiloide en la pared de arterias de pequeño y mediano calibre ${ }^{1}$. La AAC puede producir hemorragia subaracnoidea e intraparenquimatosa, produciendo depósitos de hemosiderina cerebrales, los cuales se observan en secuencias de gradiente Echo en Resonancia Magnética (RM) potenciada en T2 (T2* GE) ${ }^{2}$. Dichas secuencias son susceptibles a sustancias paramagnéticas como son, la hemosiderina y la deoxihemoglobina; por lo tanto sirve para diagnosticar, tanto hemorragias crónicas como agudas. La AAC también puede producir eventos neurológicos transitorios focales, síntomas tipo aura migrañosa, deterioro cognitivo o demencia ${ }^{2-4}$. Se han publicado episodios neurológicos transitorios debidos a microsangrados por AAC, erróneamente considerados y tratados como accidentes isquémicos transitorios (AIT) mediante tratamiento antiagregante o anticoagulante, en algunas ocasiones con consecuencias nefas$\operatorname{tas}^{3,5,6}$. Las nuevas secuencias de neuroimagen avanzada como son las potenciadas en susceptibilidad paramagnética (SWI) son capaces de detectar microsangrados cerebrales, tanto agudos como crónicos en un $25 \%$ más de los casos que en los que solo se emplean las secuencias $\mathrm{T}^{*} \mathrm{GE}^{7,8}$.

Describimos en este trabajo un caso que ilustra cómo las crisis somestésicas pueden simular AIT secundarios a microsangrados por AAC. Las RM seriadas realizadas a este paciente, incluyendo secuencias SWI, nos permitieron un seguimiento radiológico de los microsangrados secundarios a AAC.

\section{CASO CLÍNICO}

Paciente varón de 78 años de edad, con antecedentes personales de hipertrigliceridemia e hipertensión arterial, acudió al servicio de Urgencias por desarrollar afasia motora de manera súbita. El electrocardiograma (ECG) fue normal, mostrando ritmo sinusal con una frecuencia de 66 latidos por minuto y la radiografía de tórax mostró aumento de tamaño de la silueta cardíaca compatible con cardiopatía hipertensiva. La tomografía computarizada (TC) llevada a cabo 3 horas después del inicio, descartó la existencia de hemorragia intracraneal. (Fig. 1: panel superior, imagen 1). El eco-Doppler transcraneal fue normal y el eco-Doppler carotídeo mostró placas ateromatosas que producían una estenosis menor del $30 \%$ en bifurcación carotídea derecha. Se inició tratamiento con clopidogrel $75 \mathrm{mg} /$ día y atorvastatina $40 \mathrm{mg} /$ día. La RM cerebral realizada dos días después mostró un infarto cerebral isquémico en circunvolución media del lóbulo frontal izquierdo (Fig. 1: panel superior, imagen 3). En SWIRM se objetivaron depósitos de hemosiderina en los espacios subaracnoideos en ambas regiones frontales y a nivel córtico-subcortical con una distribución difusa supratentorial (Fig. 1: panel superior, imagen 2). Durante el ingreso se realizó un ecocardiograma-Doppler que objetivó un ventrículo izquierdo hipertrófico con función sistólica global normal y una monitorización ECG durante 48 horas no evidenció arritmias subclínicas.

El trastorno motor del lenguaje mejoró en las siguientes 12 semanas, permaneciendo leve déficit de la denominación. Seis meses más tarde, el paciente acudió de nuevo al Servicio de Urgencias por presentar en los dos días previos, cinco episodios de entumecimiento de la mano izquierda de unos 5-10 minutos de duración. La exploración neurológica únicamente mostró ligero déficit de denominación secundario al ictus previo. La TC cerebral reveló una tenue hiperdensidad (5x2 mm) en surco central derecho (Fig. 1: panel central, imagen 1). La RM cerebral, que incluía secuencias SWI, llevada a cabo 24 horas después del ingreso, mostró una hiposeñal en dicha región por microsangrado agudo (hallazgo no presente en secuencias SWI previas) (Fig. 1: panel medio, imagen 2). Las secuencias de RM FLAIR mostraron intensa leucoaraiosis (Fig. 1: panel central, imagen 3). La angio-RM cerebral no detectó estenosis arteriales ni presencia de aneurismas. No hubo cambios en los hallazgos del eco-Doppler transcraneal y carotídeo. En esta ocasión ni el ECG ni en la radiografía de tórax mostraron cambios respecto a estudios previos. El electroencefalograma (EEG) no mostró alteraciones. El tratamiento con clopidogrel $75 \mathrm{mg} /$ día, fue sustituido por ácido acetil salicílico $100 \mathrm{mg} /$ día debido a la necesidad de mantener antiagregación por los factores de riesgo vascular subyacentes y el antecedente de ictus isquémico. Durante las siguientes tres semanas, el paciente presentó seis nuevos episodios de 5 minutos de duración similares a los previos con la salvedad de que en esta ocasión, la hipoestesia se iniciaba en la mano izquierda pero ascendía por todo el brazo extendiéndose hasta la zona proximal de extremidad inferior ipsilateral. Una nueva TC no mostró signos de hemorragia aguda (Fig. 1: panel 
inferior, imagen 1), sin embargo, las secuencias potenciadas en T1 y FLAIR mostraron extensión medial del microsangrado previo hacia la cisura central derecha (Fig. 1: panel inferior, imágenes 2 y 3). Los episodios fueron considerados como crisis epilépticas sensitivas con propagación jacksoniana secundarias al microsangrado contralateral en el surco frontal central derecho. Se inició tratamiento con lamotrigina $100 \mathrm{mg} /$ día y la dosis de ácido acetil salicílico se redujo a $50 \mathrm{mg} /$ día. Dieciocho meses después el paciente aparece libre de episodios.

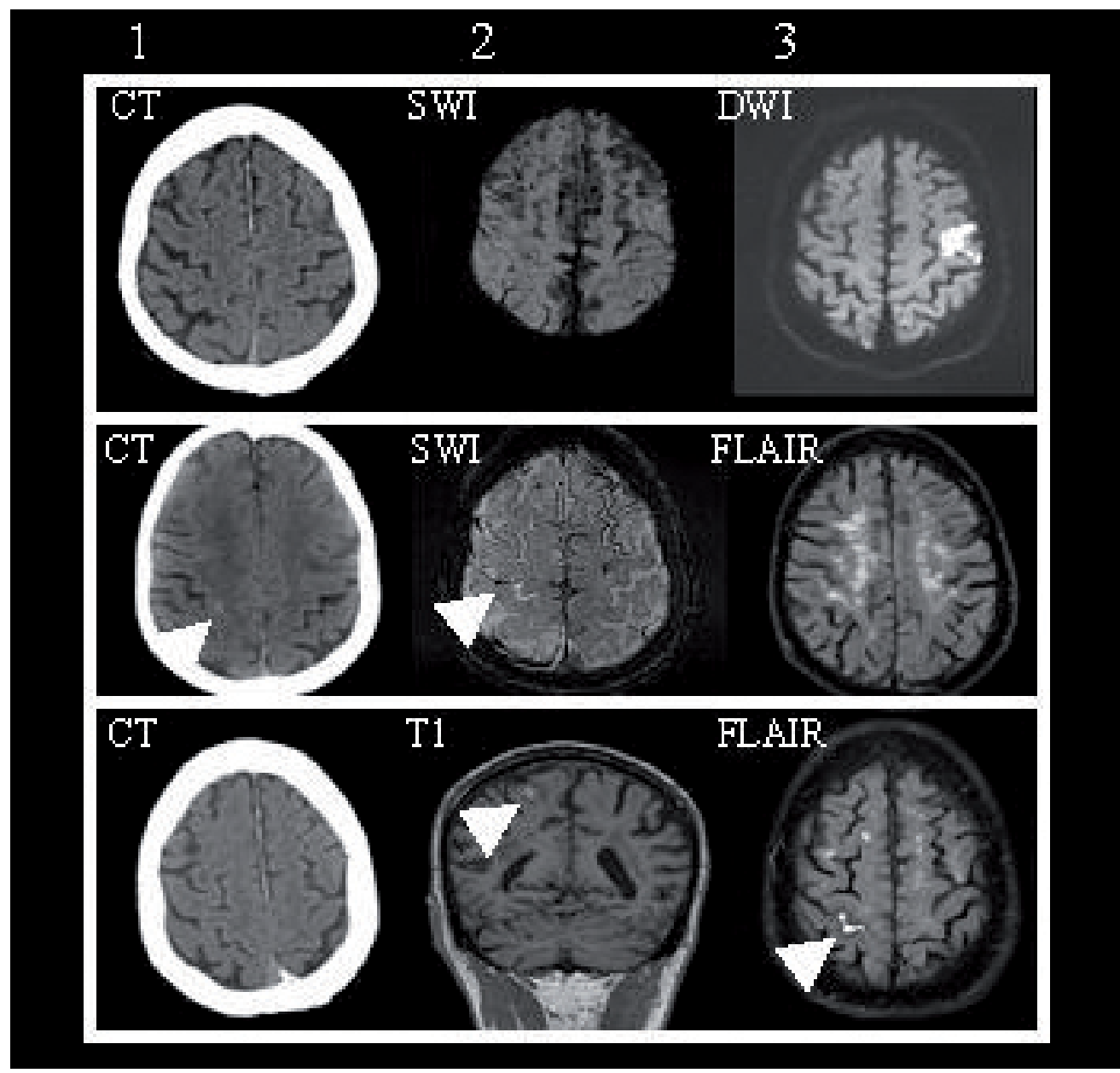

Figura 1. Imágenes de RM y TC seriadas realizadas en la primera visita (panel superior), a los 6 meses (panel central) y a los 7 meses (panel inferior).

Panel superior. La TC no muestra signos de hemorragia aguda (1). Múltiples depósitos de hemosiderina en SWI (2) y las secuencias de difusión (DWI) que muestran un infarto agudo secundario a un ictus isquémico en circunvolución media del lóbulo frontal izquierdo (3).

Panel central. La TC muestra presencia de deoxihemoglobina secundaria a microhemorragia en espacio subaracnoideo del surco central derecho (1), más clarividente en secuencias SWI (flecha blanca) (2). Leucoaraiosis extensa subcortical en secuencias FLAIR.

Panel inferior. La TC no muestra signos de sangrado (1). Las secuencias potenciadas en T1 y FLAIR muestran metahemoglobina extracelular debido a antiguo sangrado cortical, con extensión medial hacia cisura central derecha (2 y 3) (flechas blancas). 


\section{DISCUSIÓN}

Este caso indica la importancia de diferenciar entre crisis epilépticas focales y AIT en pacientes con AAC. Las crisis somestésicas aquí descritas eran clínicamente indistinguibles de AIT, pero la remisión completa tras tratamiento antiepiléptico apoya su naturaleza epiléptica ${ }^{3,6,9}$. La normalidad del EEG en crisis parciales simples, no excluye el diagnóstico de epilepsia ${ }^{10}$.

La literatura describe que algunos pacientes con episodios neurologicos transitorios asociados a AAC pueden responder al tratamiento antiepiléptico ${ }^{3,5,6,11,12}$, por lo que tenemos la impresión que los eventos transitorios en pacientes con AAC son causados por microsangrados corticales más frecuentemente de lo esperado. Además, los pacientes con AAC que reciben tratamiento antiagregante o anticoagulante, tienen potencialmente un incremento en el riesgo de complicaciones hemorrágicas recurrentes, indicando que un diagnóstico etiológico preciso inicial es crucial para una evolución clínica favorable. En caso de que las secuencias SWI de RM no hubieran estado disponibles en nuestro centro, podríamos haber diagnosticado erróneamente los episodios del paciente como AIT incrementando el tratamiento antiagregante y, probablemente, aumentando el riesgo de sangrado cerebral. Por ello, queremos resaltar, que en pacientes con AAC con síntomas focales transitorios, incluso con síntomas negativos, debemos considerar el diagnóstico de crisis epilépticas además de AIT. Las secuencias de neuroimagen avanzada, incluyendo las secuencias SWI-RM, pueden ser muy útiles para identificar microsangrados agudos o depósitos de hemosiderina debidos a sangrados por AAC. Además, en estos pacientes, el tratamiento antiepiléptico, puede ser altamente efectivo, tal y como se ha descrito , $^{3,5,6,11,12}$. Proponemos además que, en estos casos, es prudente valorar la reducción o supresión de tratamiento antiagregante para evitar ulteriores complicaciones ${ }^{6}$ dado que la AAC asociada a elevado número de microsangrados y antecedentes de accidente cerebrovascular isquémico, especialmente de distribución lobar, pueden tener mayor riesgo de sufrir una hemorragia intraparenquimatosa ${ }^{13,14}$. No obstante, son necesarios estudios prospectivos que clarifiquen el riesgo de complicaciones hemorrágicas y que establezcan la prevalencia de AAC en población adulta ${ }^{13,14}$.

\section{BIBLIOGRAFÍA}

1. VinTERS HV. Cerebral amyloid angiopathy. A critical review. Stroke 1987; 18: 311-324.

2. Greenberg SM, Finklestein SP, Schaefer PW. Petechial hemorrhages accompanying lobar hemorrhage: detection by gradient-echo MRI. Neurology 1996; 46: 1751-1754.

3. Greenberg SM, Vonsattel JP, Stakes JW, Gruber M, FinkLestein SP. The clinical spectrum of cerebral amyloid angiopathy: presentations without lobar hemorrhage. Neurology 1993; 43: 2073-2079.

4. Thanvi B, Robinson T. Sporadic cerebral amyloid angiopathy-an important cause of cerebral haemorrhage in older people. Age Ageing 2006; 35: 565-571.

5. Karabatsou K, Lecky BR, Rainov NG, Broome JC, WHITE RP. Cerebral amyloid angiopathy with symptomatic or occult subarachnoid haemorrhage. Eur Neurol 2007; 57: 103-105.

6. KleinIG TJ, Kiley M, Thompson PD. Acute convexity subarachnoid haemorrhage: a cause of aura-like symptoms in the elderly. Cephalalgia 2008; 28: 658-663.

7. HaAcke EM, Delproposto ZS, Chaturvedi S, Sehgal V, Tenzer M, NeElaValli J et al. Imaging cerebral amyloid angiopathy with susceptibilityweighted imaging. AJNR Am J Neuroradiol 2007; 28: 316-317.

8. Tong KA, Ashwal S, Holshouser BA, Shutter L, Herigault G, HaAcke EM et al. Hemorrhagic shearing lesions in children and adolescents with posttraumatic diffuse axonal injury: improved detection and initial results. Radiology $2003 ; 227: 332-339$.

9. MaIA LF, Mackenzie IR, Feldman HH. Clinical phenotypes of cerebral amyloid angiopathy. J Neurol Sci 2007; 257: 23-30.

10. Devinsky O, Kelley K, Porter RJ, Theodore WH. Clinical and electroencephalographic features of simple partial seizures. Neurology 1988; 38: 1347-1352.

11. Izenberg A, Aviv RI, Demaerschalk BM, DoDICK DW, Hopyan J, BLACK SE et al. Crescendo transient Aura attacks: a transient ischemic 
attack mimic caused by focal subarachnoid hemorrhage. Stroke 2009; 40: 3725-3729.

12. Sмiтh DB, Нiтchсоск M, Philpotт PJ. Cerebral amyloid angiopathy presenting as transient ischemic attacks. Case report. J Neurosurg 1985; 63: 963-964.

13. Soo YO, Yang SR, Lam WW, Wong A, Fan YH, LEUNG HH et al. Risk vs benefit of anti-thrombotic therapy in ischaemic stroke patients with cerebral microbleeds. J Neurol 2008; 255: 1679-1686.
14. Gregoire SM, Jager HR, Yousry TA, Kallis C, Brown MM, WerRING DJ. Brain microbleeds as a potential risk factor for antiplatelet-related intracerebral haemorrhage: hospital-based, case-control study. J Neurol Neurosurg Psychiatry 2010; 81: 679-684. 
